\title{
FUNGOS MICORRÍZICOS ARBUSCULARES EM CAMPOS DE MURUNDUS APÓS A CONVERSÃO PARA SISTEMAS AGRÍCOLAS NO CERRADO(1)
}

\author{
Paula Camylla Ramos Assis ${ }^{(2)}$, Orivaldo José Saggin Júnior ${ }^{(3)}$, Helder Barbosa Paulino ${ }^{(4)}$, \\ Sidney Luiz Stürmer ${ }^{(5)}$, José Oswaldo Siqueira ${ }^{(6)} \&$ Marco Aurélio Carbone Carneiro( $^{(7)}$
}

\section{RESUMO}

\begin{abstract}
Os campos de murundus são fitofisionomias de ocorrência no bioma Cerrado com funções ecológicas importantes para a manutenção da sustentabilidade do solo; e a conversão para sistemas agrícolas pode provocar alterações nos atributos físicos, químicos e biológicos do solo ainda não avaliados, como a redução da biodiversidade de fungos micorrízicos arbusculares. O objetivo deste estudo foi avaliar como a conversão dos campos de murundus em áreas de sistema agrícola altera a comunidade de fungos micorrízicos arbusculares (FMAs). Os tratamentos constituíram-se de três áreas agrícolas submetidas ao mesmo manejo e uso agrícola em uma cronossequência (7, 11 e 14 anos) e duas na área referência [campo de murundus, em topo (TM) e entre os murundus (EM)]. Os esporos de FMAs foram extraídos, contados, e as espécies de FMAs identificadas pelas características morfológicas. $\mathrm{O}$ total de FMAs recuperado foi de 27 espécies, sendo nove espécies da família Acaulosporaceae, uma Ambisporaceae, sete Glomeraceae, duas Claroideoglomeraceae e oito Gigasporaceae. Desse total, as espécies Acaulospora scrobiculata, Glomus macrocarpum, e Gigaspora sp. ocorreram em todas as áreas nos dois anos estudados. As espécies Acaulospora mellea, Acaulospora cavernata, Acaulospora colombiana, Glomus diaphanum, Scutellospora reticulata e Scutellospora sp. só foram encontradas nos campos de murundus. A conversão de campos de murundus em área agrícola modificou a ocorrência e composição da comunidade de FMAs; as espécies Acaulospora scrobiculata, Glomus macrocarpum, Claroideoglomus etunicatus e Gigaspora sp
\end{abstract}

(1) Parte da Dissertação de Mestrado da primeira autora apresentada ao Programa de Pós-Graduação em Agronomia, Universidade Federal de Goiás - Regional Jataí. Recebido para publicação em 16 de abril de 2014 e aprovado em 21 de agosto de 2014.

(2) Doutoranda do Programa de Pós-Graduação em Agronomia, Universidade Federal de Goiás - UFG. Campus Samambaia. Rod. Goiânia - Nova Veneza, km 0. CEP 740001-970 Goiânia (GO). E-mail: paulacamyllaramos@gmail.com

(3) Pesquisador A, Embrapa Agrobiologia. BR 465, km 7. CEP 23890-000 Seropédica (RJ). E-mail: orivaldo.saggin@embrapa.br

(4) Professor Associado, Universidade Federal de Goiás, Regional de Jataí, Laboratório de Solos. BR 364, km 192. CEP 75804-020. Jataí (GO). E-mail: helderlino51@yahoo.com.br

(5) Professor Associado, Departamento de Ciências Naturais, Universidade Regional de Blumenau. Rua Antônio da Veiga, 140. Caixa Postal 1507. CEP 89012-900 Blumenau (SC). Bolsista do CNPq. E-mail: sturmer@furb.br

(6) Professor Emérito, Instituto Tecnológico Vale. Rua Boaventura da Silva, 955, Nazaré. CEP 60055-090 Belem (PA). Bolsista do CNPq. E-mail: jose.oswaldo.siqueira@itv.org

(7) Professor Associado, Departamento de Ciência do Solo, Universidade Federal de Lavras. Caixa Postal 3037. CEP 37200-000 Lavras (MG). Bolsista do CNPq. E-mail: marcocarbone@dcs.ufla.br 
ocorreram em todas as áreas e a não ocorrência de algumas espécies nas áreas de cultivo, como as espécies Acaulospora cavernata, Acaulospora colombiana, Rhizophagus diaphanus, Scutellospora reticulata e Scutellospora sp. representa perda de diversidade desses fungos. Portanto, este estudo tratou-se do primeiro relato da ocorrência e da estrutura da comunidade de FMAs em fitofisionomia de campos de murundus, contribuiu para o maior entendimento dos FMAs no bioma Cerrado e demonstrou que as alterações promovidas pela conversão da área alteraram a ocorrência e a diversidade dos fungos micorrízicos arbusculares.

Termos de indexação: biodiversidade de FMAs, plantio direto, áreas alagadas.

\title{
SUMMARY: ARBUSCULAR MYCORRHIZAL FUNGI IN "MURUNDU FIELDS" AFTER CONVERSION TO FARM SYSTEMS IN THE CERRADO
}

\begin{abstract}
The murundu fields (Campos de Murundus) are characterized by mounds occurring in some parts of the Cerrado (Brazilian tropical savanna) biome with important ecological functions for the maintenance of soil sustainability. Conversion to agricultural systems may lead to changes in physical, chemical, and biological soil properties not yet assessed, such as reduction in the biodiversity of arbuscular mycorrhizal fungi. The aim of this study was to evaluate how the conversion of mound fields into agricultural areas changes the community of arbuscular mycorrhizal fungi $(A M F)$. Treatments consisted of three agricultural areas subjected to the same agricultural management and use in a chronosequence (seven, 11, and 14 years) and two in the mound fields, sampling at the top (TM) and between the mounds (EM)). AMF spores were extracted and counted, and AMF species were identified by morphological characteristics. The total AMF recovered was 27 species, made up of nine species of the Acaulosporaceae family, one Ambisporaceae, seven Glomeraceae, two Claroideoglomeraceae, and eight Gigasporaceae. Of this total, the species Acaulospora scrobiculata, Glomus macrocarpum, and Gigaspora $s p$ occurred in all areas in the two years studied. The species Acaulospora mellea, Acaulospora cavernata, Acaulospora colombiana, Glomus diaphanum, Scutellospora reticulata, and Scutellospora sp only occurred in the mound fields. The conversion of mound fields into an agricultural area modified the occurrence and composition of the arbuscular mycorrhizal fungi community, and the species Acaulospora scrobiculata, Glomus macrocarpum, Gigaspora $s p$, and Claroideoglomus etunicatus occurred in all areas. The non-occurrence of some species in the crop areas, such as the species Acaulospora cavernata, Acaulospora columbiana, Rhizophagus diaphanus, Scutellospora reticulata, and Scutellospora sp, represents a loss of diversity of these fungi. Therefore, this study is the first report of the occurrence and community structure of $A M F$ in the mound field vegetation type. It contributes to better understanding of AMF in the Cerrado biome and shows that the changes introduced by conversion of the area alter the occurrence and diversity of arbuscular mycorrhizal fungi.
\end{abstract}

Index terms: AMF biodiversity, no tillage, flooded areas.

\section{INTRODUÇÃO}

O Cerrado brasileiro destaca-se entre os biomas tropicais por apresentar grande potencial agrícola e produtor de grãos, carne, fibras e, mais recentemente, de energia (biocombustíveis). Isso deve-se, em grande parte, à concentração de solos com boa aptidão agrícola como os Latossolos, que representam de 50 a $60 \%$ da área desse bioma (Souza \& Lobato, 2004). No entanto, em razão da expansão agrícola e da forte pressão do agronegócio, foram incorporadas ao sistema de produção áreas que possuem solos com baixa aptidão agrícola como os solos hidromórficos, que em algumas regiões do Brasil Central estão associados a uma fitofisionomia típica, denominada "Campos de Murundus".

Esses campos apresentam montículos de solo, denominados de murundus, de formato circular variando 2 a $10 \mathrm{~m}$ de diâmetro e até $2 \mathrm{~m}$ de altura no Brasil, podendo chegar até $10 \mathrm{~m}$ de altura, como encontrado na África (Cox et al., 1989), sendo formado provavelmente pela ação de térmitas. O solo apresenta baixa capacidade de infiltração de água permanecendo inundado entre os murundus no período das chuvas, sendo vegetado por espécies tolerantes ao alagamento temporário. O terço superior dos murundus, parte seca do solo, apresenta vegetação típica do Cerrado strictu sensu, composta por espécies arbustivas e arbóreas (Resende et al., 2007; Marimon et al., 2012). Os campos de murundus também são encontrados na África, Austrália e América do Norte (Mollard, 1982; Cox et al., 1989; Rahlao et al., 2008) e, no Brasil, no bioma de Cerrado (Oliveira Filho, 1992; Silva et al., 2010).

Para a conversão dessas áreas em solo agrícola, há necessidade de construção de canais de drenagem, 
nivelamento do terreno com a remoção dos murundus, homogeneização do solo, correção e adubação do solo, promovendo alterações nos atributos físicos, químicos e possivelmente os biológicos; no entanto, não há pesquisa sobre essas alterações e suas consequências.

Os fungos micorrízicos arbusculares (FMAs) por formarem associações simbióticas com as raízes da maioria das plantas vasculares, promovendo melhorias na absorção de nutrientes, adaptação a estresses bióticos e abióticos do solo, entre outros benefícios à comunidade vegetal, possuem relação direta com diversidade de plantas em determinados ecossistemas (van der Heijden et al., 1998; Klironomos et al., 2000). Portanto, FMAs são componentes importantes em ambientes naturais e também nos alterados pelo homem como nos solos agrícolas, podendo ser utilizados como bioindicadores de qualidade do solo. Vários trabalhos referem-se à comunidade dos FMAs no bioma Cerrado (Siqueira et al., 1989; Cordeiro et al., 2005; Miranda et al., 2005; Ferreira et al., 2012), no entanto, não foram encontrados registros de estudos desses fungos em campos de murundus.

Assim, a conversão de campos de murundus em sistemas agrícolas pode proporcionar situação interessante para avaliar a interferência antrópica na ocorrência e estrutura da comunidade dos FMAs. Nesse sentido, o objetivo deste estudo foi avaliar como a conversão dos campos de murundus em áreas de cultivo altera a comunidade de fungos micorrízicos arbusculares.

\section{MATERIAL E MÉTODOS}

A área estudada localiza-se a $17^{\circ} 57^{\prime} 11^{\prime \prime} \mathrm{S}$ e $52^{\circ} 04^{\prime} 45^{\prime \prime} \mathrm{O}$ com altitude de $872 \mathrm{~m}$, entre os municípios de Jataí e Mineiros, GO. O clima da região é classificado segundo Köppen como Cw, mesotérmico, com estação seca (maio a setembro) e chuvosa (outubro a abril). O solo da área estudada foi classificado como Plintossolo Háplico, apresentando condições de restrição à percolação da água e ao efeito temporário de alagamento por um período de quatro a seis meses com formação de plintita, em profundidade superior a $60 \mathrm{~cm}$.

O estudo constou de uma cronossequência de conversão do campo de murundus em áreas agrícolas (7, 11 e 14 anos de uso agrícola, referente a 2011). A conversão dos campos de murundus em solos cultivados iniciou-se com a queima da vegetação seguida do preparo do solo com grade aradora e niveladora. Os murundus presentes foram escarificados/subsolados; em seguida, terraplanados com lâminas frontais e de arrasto, removendo o solo para entre os murundus em processos sucessivos até o completo aplainamento do terreno. Para evitar o alagamento, comum nessa área, foi construída uma rede de drenos com espaçamento entre os canais de cerca de $100 \mathrm{~m}$ e profundidade variando de 0,5 a $2 \mathrm{~m}$.

Nessas áreas cultivadas, os manejos do solo e das culturas foram semelhantes, utilizando a sucessão soja na safra e milho na safrinha, em sistema de plantio direto; portanto, a principal variação entre essas foi o tempo de conversão. O histórico do manejo do solo e das culturas das áreas cultivadas encontra-se no quadro 1. A análise textural verificou 445, $25 \mathrm{e}$ $530 \mathrm{~g} \mathrm{~kg}^{-1}$ de argila, silte e areia, respectivamente, e os resultados da caracterização química do solo encontram-se no quadro 2.

Além das áreas cultivadas, utilizou-se, como referência, área de campo de murundus sem interferência antrópica, considerando duas situações distintas, o topo dos murundus (TM) e entre os murundus (EM). Essa diferenciação deve-se em razão da diferença na composição da vegetação e do alagamento que ocorre entre essas posições nos murundus.

Em cada uma das áreas estudadas, foram demarcados em torno de 65 quadrados de $100 \times 100 \mathrm{~m}$. Desses, foram sorteados 10 quadrados aleatórios (parcelas), considerados como repetições dentro de cada área estudada. No interior dessas parcelas, em ziguezague, foram retiradas 10 subamostras para perfazer uma amostra composta por parcela. Na área sem interferência, foram sorteados 10 murundus aleatórios (parcelas), considerados como repetições, sendo realizada a coleta no terço superior no topo dos murundus (TM) e entre os murundus (EM).

As amostragens foram realizadas em dois anos consecutivos, em setembro de 2009 e 2010, no período seco. No momento das amostragens, os resíduos vegetais superficiais não decompostos foram retirados e coletados $2 \mathrm{~kg}$ de solo por parcela na profundidade de $0-20 \mathrm{~cm}$. As amostras de solos foram encaminhadas imediatamente para o Laboratório de Solos da UFG/ Campus Jataí e peneiradas (malha de $2 \mathrm{~mm}$ ), para retirada de raízes e pequenos organismos da fauna do solo, sendo posteriormente armazenadas a $4^{\circ} \mathrm{C}$ até o momento da análise.

Os esporos de FMAs foram extraídos de uma alíquota de $50 \mathrm{~g}$ de solo de cada amostra, conforme a técnica de Gerdemann \& Nicolson (1963), e centrifugados em gradiente de sacarose (Jenkins, 1964). Após esse procedimento, esses esporos foram contados com o auxílio de um microscópio estereoscópico com aumento de 40× e separados morfologicamente para preparo de lâminas para microscopia. Para auxiliar a identificação, foram utilizadas descrições originais das espécies, além de descrições das espécies fornecidas pelas páginas do INVAM (International Culture Collection of Arbuscular and Vesicular-Arbuscular Mycorrhizal Fungi, Morgantown, WV, EUA - http://invam.caf.wvu.edu/).

As espécies de FMA foram classificadas quanto a sua presença nas áreas estudadas em Generalistas 
(ocorrendo nas cinco áreas), Intermediárias (ocorrendo em duas a quatro áreas) e Exclusivas (ocorrendo em apenas uma área), segundo a proposta de Zhang et al. (2004).

Por se tratar de um estudo ecológico e estar localizado em um mesmo tipo de solo, em áreas contíguas, relevo plano e com o mesmo manejo do solo e de cultivo, optou-se por casualizar os pontos de amostragem dentro de cada área sorteando as parcelas (10 pseudorrepetições) e considerando como um delineamento inteiramente casualizado. Para comparação das comunidades de FMAs entre as áreas,

\section{Quadro 1. Identificação e histórico do manejo e uso das áreas estudadas}

\begin{tabular}{|c|c|}
\hline Identificação & Histórico \\
\hline $\begin{array}{l}\text { Topo (TM) e entre } \\
\text { os murundus (EM) }\end{array}$ & $\begin{array}{l}\text { Área sem intervenção antrópica. Os murundus apresentam-se com diâmetro médio de } \\
6 \mathrm{~m} \text { e altura em torno de } 1 \text { a } 2 \mathrm{~m} \text {. A parte superior dos murundus (TM) apresenta vegetação } \\
\text { típica de Cerrado stritus sensu, com elevada diversidade de plantas arbustivas, arbóreas e rasteiras } \\
\text { e constante presença de cupins. A coleta de solo foi realizada no terço superior dos murundus. } \\
\text { Entre os murundus (EM), há ocorrência de uma lâmina de água em torno de } 10 \mathrm{~cm} \text { de altura no } \\
\text { período chuvoso e presença de espécies vegetais do gênero Syngonanthus sp., tolerantes a esse } \\
\text { alagamento. Possui em torno de } 148 \text { ha com } 33 \text { murundus. }\end{array}$ \\
\hline Área 14 anos & $\begin{array}{l}\text { Área que sofreu intervenção antrópica desde 1996/1997. Inicialmente, apresentava-se sob } \\
\text { pastagem nativa degradada, que em } 1996 \text { aplicaram-se } 3 \mathrm{t} \mathrm{ha}^{-1} \text { de calcário dolomítico com a } \\
\text { incorporação, utilizando arado e grade niveladora. No plantio inicial, aplicaram-se } 1 \mathrm{t} \mathrm{ha}^{-1} \text { fosfato } \\
\text { reativo ( } 33 \% \mathrm{P}_{2} \mathrm{O}_{5} \text { ) e } 2 \mathrm{t} \mathrm{ha}^{-1} \text { de gesso. A partir de } 1998 \text {, não houve revolvimento do solo, ou seja, } \\
\text { utilizou-se plantio direto. Em } 2005 / 2006 \text {, aplicou-se } 1,5 \mathrm{t} \mathrm{ha}^{-1} \text { de calcário dolomítico superficialmente. } \\
\text { Nessa área, a sucessão de cultura realizada foi com soja na safra e milho na safrinha, obtendo- } \\
\text { se produtividade em torno de } 3,4 \text { e } 6 \mathrm{t} \mathrm{ha}^{-1} \text {, respectivamente. }\end{array}$ \\
\hline Área 11 anos & $\begin{array}{l}\text { Área que sofreu intervenção antrópica desde 1999/2000. Inicialmente, apresentava-se sob áreas } \\
\text { nativas e pastagem nativa, que em } 1999 \text { aplicaram-se } 6 \mathrm{t} \text { ha }{ }^{-1} \text { de calcário dolomítico com a } \\
\text { incorporação, utilizando arado e grade niveladora. No plantio inicial, aplicou-se } 0,6 \mathrm{t} \mathrm{ha}^{-1} \text { de } \\
\text { fosfato reativo }\left(33 \% \mathrm{P}_{2} \mathrm{O}_{5} \text { ). A partir de } 2000 \text {, não houve revolvimento do solo, ou seja, utilizou- }\right. \\
\text { se plantio direto. Nessa área, a sucessão de cultura realizada foi com soja na safra e milho na } \\
\text { safrinha, obtendo-se produtividade em torno de } 3,4 \text { e } 6 \mathrm{t} \mathrm{ha}^{-1} \text {, respectivamente, nos primeiros } \\
\text { anos; a partir de } 2006 \text {, soja/pousio. Em } 2002 / 2003 \text { e } 2007 / 2008 \text {, aplicaram-se } 2,5 \text { t ha }^{-1} \text { calcário } \\
\text { dolomítico superficialmente. }\end{array}$ \\
\hline Área 7 anos & $\begin{array}{l}\text { Área que sofreu intervenção antrópica desde } 2003 / 2004 \text {. Inicialmente, apresentava-se sob } \\
\text { pastagem nativa. Em } 2003 \text {, aplicaram-se } 5 \mathrm{t} \mathrm{ha}^{-1} \text { de calcário dolomítico com a incorporação, } \\
\text { utilizando arado e grade niveladora. No plantio inicial, aplicaram-se } 0,6 \mathrm{t} \mathrm{ha}^{-1} \text { de fosfato reativo } \\
\left(33 \% \mathrm{P}_{2} \mathrm{O}_{5}\right) \text { e } 2 \mathrm{t} \mathrm{ha}^{-1} \text { gesso agrícola. A partir de } 2004 \text {, não houve revolvimento do solo, ou seja, } \\
\text { utilizou-se plantio direto. Nessa área, a sucessão de cultura realizada foi com soja na safra e } \\
\text { milho (ou milheto ou sorgo) na safrinha, obtendo-se produtividade em torno de } 3,1 \text { para a soja e } \\
\text { de } 4,5 \mathrm{t} \mathrm{ha}^{-1} \text { para o milho, nos primeiros anos; a partir de } 2007 \text {, rotação soja e milho na safra e } \\
\text { pousio na entressafra. Em } 2005 / 2006 \text { e em } 2008 / 2009 \text {, aplicou-se } 1,5 \mathrm{t} \mathrm{ha}^{-1} \text { calcário dolomítico } \\
\text { superficialmente. }\end{array}$ \\
\hline
\end{tabular}

Quadro 2. Atributos químicos do solo das áreas agrícolas e do topo (MT) e entre os murundus (EM)

\begin{tabular}{|c|c|c|c|c|c|c|c|}
\hline Ano/área & $\mathrm{pH}\left(\mathrm{H}_{2} \mathrm{O}\right)^{(1)}$ & $\mathrm{Al}^{3+}$ & $\mathrm{Ca}^{2+}$ & $\mathrm{Mg}^{2+}$ & $\mathbf{K}$ & $\mathbf{P}$ & MOS \\
\hline & & \multicolumn{3}{|c|}{$-\mathrm{cmol}_{\mathrm{c}} \mathrm{dm}^{-3}$} & \multicolumn{2}{|c|}{$-\mathrm{mg} \mathrm{dm}{ }^{-3}$} & $\mathrm{~g} \mathrm{~kg}^{-1}$ \\
\hline 7 & 6,10 & 0,06 & 2,49 & 0,90 & 40,8 & 3,09 & 35,17 \\
\hline 11 & 5,84 & 0,07 & 2,76 & 1,22 & 30,5 & 3,86 & 35,18 \\
\hline 14 & 6,28 & 0,05 & 3,43 & 1,34 & 111,4 & 2,31 & 41,11 \\
\hline TM & 5,04 & 0,82 & 0,04 & 0,22 & 24,9 & 0,40 & 37,19 \\
\hline EM & 5,40 & 0,29 & 0,05 & 0,23 & 19,8 & 0,40 & 39,76 \\
\hline
\end{tabular}

(1) pH em água, relação 2:1; $\mathrm{Al}^{3+}, \mathrm{Mg}^{2+} \mathrm{e} \mathrm{Ca}^{2+}$, extraídos por $\mathrm{KCl} 1$ mol L-1; K e P, extraídos por Mehlich-1; e MOS: matéria orgânica do solo, oxidada com dicromato de potássio, em meio ácido. 
a densidade de esporos, a riqueza de espécies e os índices de diversidade, foi realizada análise de variância, e quando significativo utilizou-se o teste Scott-Knott (5\%) para diferenciação dos tratamentos, utilizando programa estatístico Sisvar (Ferreira, 2000).

\section{RESULTADOS E DISCUSSÃO}

Neste estudo, foram recuperadas 27 espécies de fungos micorrízicos arbusculares (FMAs) pertencentes a oito gêneros e cinco famílias, considerando os dois anos de pesquisa (Quadro 3). As famílias mais representativas em relação ao número de espécies recuperadas foram Acaulosporaceae (Acaulospora), Gigasporaceae (Gigaspora, Scutellospora) e Glomeraceae (Glomus, Funneliformis, Rhizophagus), com nove, oito e sete espécies de FMAs, respectivamente. Nas famílias Ambisporaceae (Ambispora) e Claroideoglomeraceae (Claroideoglomus) foram recuperadas somente uma e duas espécies de FMAs, respectivamente. Assim, como verificado por Carvalho et al. (2012), os quais recuperaram 23 espécies de FMAs na serra do Cipó, em Minas Gerais, os campos de murundus também podem ser considerados como uma região de reserva da biodiversidade hotpots.

Considerando a compilação realizada por Souza et al. (2010), para a região de Cerrado no Brasil, oito

Quadro 3. Ocorrência de famílias e espécies e classificação dos fungos micorrízicos arbusculares recuperados na cronossequência de uso agrícola $(7,11$ e 14 anos) e nas áreas sem alteração antrópica, no topo dos murundus (TM) e entre os murundus (EM)

\begin{tabular}{|c|c|c|c|c|c|c|}
\hline Família/Espécie & 7 anos & 11 anos & 14 anos & TM & EM & Classif. $^{(1)}$ \\
\hline \multicolumn{7}{|l|}{ Família Acaulosporaceae } \\
\hline Acaulospora cavernata Blaszkowski & 0 & 0 & 0 & 3 & 3 & $\mathrm{E}$ \\
\hline Acaulospora denticulata Sieverding \& Toro & 11 & 2 & 5 & 1 & 0 & I \\
\hline Acaulospora foveata Trappe \& Janos & 9 & 10 & 3 & 4 & 0 & I \\
\hline Acaulospora mellea Spain \& Schenck & 0 & 0 & 0 & 2 & 1 & I \\
\hline Acaulospora rehmii Sieverding \& Toro & 0 & 0 & 2 & 1 & 0 & I \\
\hline Acaulospora scrobiculata Trappe & 12 & 13 & 11 & 5 & 9 & $\mathrm{G}$ \\
\hline Acaulospora tuberculata Janos \& Trappe & 2 & 2 & 0 & 3 & 2 & I \\
\hline Acaulospora undulata Sieverding & 0 & 1 & 0 & 0 & 0 & $\mathrm{E}$ \\
\hline Acaulospora colombiana (Spain \& Schenck) Kaonongbua, Morton \& Bever & 0 & 0 & 0 & 2 & 0 & $\mathrm{E}$ \\
\hline \multicolumn{7}{|l|}{ Família Ambisporaceae } \\
\hline Ambispora leptoticha (Schenck \& Smith) Walker, Vestberg \& Schüßler & 1 & 0 & 1 & 1 & 1 & I \\
\hline \multicolumn{7}{|l|}{ Família Glomeraceae } \\
\hline Glomus macrocarpum Tulasne \& Tulasne & 7 & 9 & 10 & 14 & 12 & $\mathrm{G}$ \\
\hline Glomus tortuosum Schenck \& Smith & 3 & 3 & 2 & 0 & 0 & $\mathrm{I}$ \\
\hline Glomus clavispora Trappe & 7 & 1 & 2 & 1 & 4 & $\mathrm{G}$ \\
\hline Glomus sp. & 0 & 2 & 0 & 0 & 0 & $\mathrm{E}$ \\
\hline Rhizophagus clarus ( Nicolson \& Schenck) Walker \& Schüßler & 3 & 2 & 2 & 1 & 4 & G \\
\hline Rhizophagus diaphanus (Morton \& Walker) Walker \& Schüßler & 0 & 0 & 0 & 2 & 0 & $\mathrm{E}$ \\
\hline Funneliformis geosporus (Nicolson \& Gerdemann) Walker \& Schüßler & 2 & 1 & 0 & 0 & 0 & I \\
\hline \multicolumn{7}{|l|}{ Família Claroideoglomeraceae } \\
\hline Claroideoglomus etunicatum (Becker \& Gerdemann) Walker \& Schüßler & 3 & 6 & 4 & 6 & 4 & $\mathrm{G}$ \\
\hline Claroideoglomus lamellosum (Dalpé, Koske \& Tews) Walker \& Schüßler & 1 & 2 & 0 & 0 & 0 & I \\
\hline \multicolumn{7}{|l|}{ Família Gigasporaceae } \\
\hline Gigaspora gigantea (Nicolson \& Gerdemann) Gerdemann \& Trappe & 1 & 0 & 0 & 0 & 0 & $\mathrm{E}$ \\
\hline Gigaspora sp & 8 & 10 & 9 & 8 & 3 & G \\
\hline Scutellospora heterogama (Nicolson \& Gerdemann) Walker \& Sanders & 3 & 3 & 1 & 1 & 0 & I \\
\hline Scutellospora nigra (Redhead) Walker \& Sanders & 2 & 0 & 0 & 1 & 1 & I \\
\hline Scutellospora pellucida (Nicolson \& Schenck) Walker \& Sanders & 2 & 5 & 10 & 3 & 0 & I \\
\hline Scutellospora reticulata (Koske, Miller \& Walker) Walker \& Sanders & 0 & 0 & 0 & 2 & 0 & $\mathrm{E}$ \\
\hline Scutellospora scutata Walker \& Diederich & 0 & 5 & 4 & 3 & 1 & $\mathrm{I}$ \\
\hline Scutellospora sp. & 0 & 0 & 0 & 1 & 0 & $\mathrm{E}$ \\
\hline
\end{tabular}

(1) Classificação adaptada de Zhang et al. (2004), em que G são espécies Generalistas que ocorreram em todas a áreas; I, são Intermediárias que ocorreram em duas a quatro áreas; e E, são Exclusivas que ocorreram em somente uma área. 
dessas espécies (Acaulospora cavernata, Acaulospora denticulata, Acaulospora foveata, Acaulospora mellea, Acaulospora rehmii, Ambispora leptoticha, Scutellospora scutata e Glomus tortuosum) representam o primeiro registro para o bioma Cerrado e o primeiro estudo referente aos campos de murundus e em áreas cultivadas adjacentes.

Das oito espécies classificadas como de ocorrência exclusiva, cinco delas somente foram registradas em campo de murundus (Acaulospora cavernata, Acaulospora colombiana, Rhizophagus diaphanus, Scutellospora reticulata e Scutellospora sp.) e as espécies Acaulospora undulata, Glomus tortuosum, Glomus sp., Funneliformis geosporus, Claroideoglomus lamellosum e Gigaspora gigantea foram recuperadas somente nas áreas cultivadas. Estudos em outros agroecossistemas no bioma Cerrado do Brasil demonstram redução do número de espécies de FMAs recuperadas sob intervenção antrópica (Cordeiro et al., 2005; Miranda, 2008; Ferreira et al., 2012), o que pode comprometer o desenvolvimento das plantas micotróficas nessas áreas. Já neste estudo os resultados evidenciam que a conversão de área nativa de campo de murundus em sistemas agrícolas resulta em modificações e mudanças na composição da comunidade de FMAs.

Apenas três espécies de FMAs (Acaulospora scrobiculata, Glomus macrocarpum e Claroideoglomus etunicatum) foram recuperadas em todas as áreas estudadas. As espécies pertencentes à família Glomeraceae foram consideradas, em sua maioria, generalistas, o que pode ser por causa da sua grande capacidade de esporulação e da alta adaptabilidade a solos agrícolas, como também observados em outros trabalhos (Siqueira et al., 1989; Cordeiro et al., 2005; Ferreira et al., 2012).

Stürmer \& Siqueira (2011), ao estudarem a alteração promovida pelo manejo e uso do solo na comunidade de espécies de FMAs na Amazônia Brasileira, verificaram que $84 \%$ de todas as espécies recuperadas de FMAs pertenciam aos gêneros Acaulospora e Glomus e notaram que padrão semelhante foi observado em outros sistemas tropicais, como a China, Panamá, Brasil e México (Zhao et al., 2003; Mangan et al., 2004; Zhang et al., 2004; Stürmer \& Siqueira, 2006; Guadarrama-Chávez et al., 2007; Zangaro et al., 2007). Neste estudo, esses dois gêneros compõem a maior fração da diversidade de espécies, compreendendo mais de $70 \%$ da diversidade total e confirmam a capacidade desses gêneros de adaptação às condições adversas.

$\mathrm{O}$ pH do solo e o teor de $\mathrm{Al}$ têm sido dois dos principais fatores edáficos que regulam a composição das comunidades de FMA (Sieverding, 1991; Correia et al., 2004); no entanto, a correção desse solo com aplicação de calcário, aliado à fertilização, principalmente fosfatados solúveis, promoveram pequena alteração do número de espécies de FMAs recuperadas em áreas cultivadas. A exceção para
Acaulospora colombiana, que foi recuperada somente no topo dos murundus, onde o pH está em torno de 5,0. Segundo Sieverding (1991), com base em vários levantamentos feitos em diferentes regiões tropicais, observou-se que essa espécie não ocorre em solos com o pH acima de 5,0, o que corrobora esta pesquisa.

A riqueza total de espécies de FMAs recuperadas, considerando os dois anos estudados, reduziu com o aumento da idade de conversão de campos de murundus para sistemas agrícolas, sendo recuperadas 17, 16 e 14 espécies de FMAs nas áreas de sete, 11 e 14 anos, respectivamente, diferindo significativamente do obtido no topo dos murundus (Figura 1a). As práticas agrícolas como o preparo do solo e a adição de fertilizantes químicos, principalmente $\mathrm{P}$, causam efeitos diretos sobre as populações de FMAs nativas, interferindo também na estrutura da comunidade micorrízica com a consequente redução na riqueza específica (Siqueira et al., 1990; Carrenho et al. 2001; Miranda \& Miranda, 2007). Esse comportamento também foi encontrado neste estudo, em que se verificou redução na riqueza específica das áreas de sistema agrícolas em relação à área de topo dos murundus (TM), o que pode ser atribuído ao impacto do sistema agrícola.

Já na área entre os murundus (EM), foram recuperadas, no total, 12 espécies de FMAs. A diferença espacial entre o topo (TM) e entre os murundus (EM) refere-se ao alagamento que acontece no periódico chuvoso. Vários estudos demonstraram que o alagamento reduz a densidade de esporos, colonização micorrízica e riqueza de FMAs (Carvalho et al., 2001; Entry et al., 2002; Escudero \& Mendonza, 2005; Wang et al., 2010; Stevens et al., 2011), corroborando os resultados.

As áreas de 7 anos e o topo dos murundus (TM) apresentaram a maior densidade de esporos, diferindo significativamente $(\mathrm{p} \leq 0,05)$ das demais áreas estudadas (Figura 1b). Várias pesquisas ressaltaram que em áreas cultivadas a densidade de esporos recuperados foi maior em comparação às áreas sob vegetação natural (Siqueira et al., 1989; Cordeiro et al., 2005; Miranda et al., 2005; Ferreira et al., 2012). Miranda \& Miranda (1997) verificaram em solos do Cerrado que a população de FMAs é geralmente baixa e pode aumentar gradativamente com a correção do solo e o cultivo de plantas micotróficas, como é o caso da soja, do milho e da braquiária. A mudança do uso do solo alterou a ocorrência das espécies de FMAs e o efeito dessa alteração na comunidade ainda precisa ser estudada em relação à redução da sustentabilidade e da própria resiliência do solo.

Espécies ou morfotipos de FMAs, como espécies raras, espécies ainda não identificadas e, ou, endêmicas da fitofisionomia de campos de murundus, como as exclusivas apresentadas nesta pesquisa, podem estar sendo perdidas antes que a evolução da taxonomia molecular desvende sua real diversidade e 


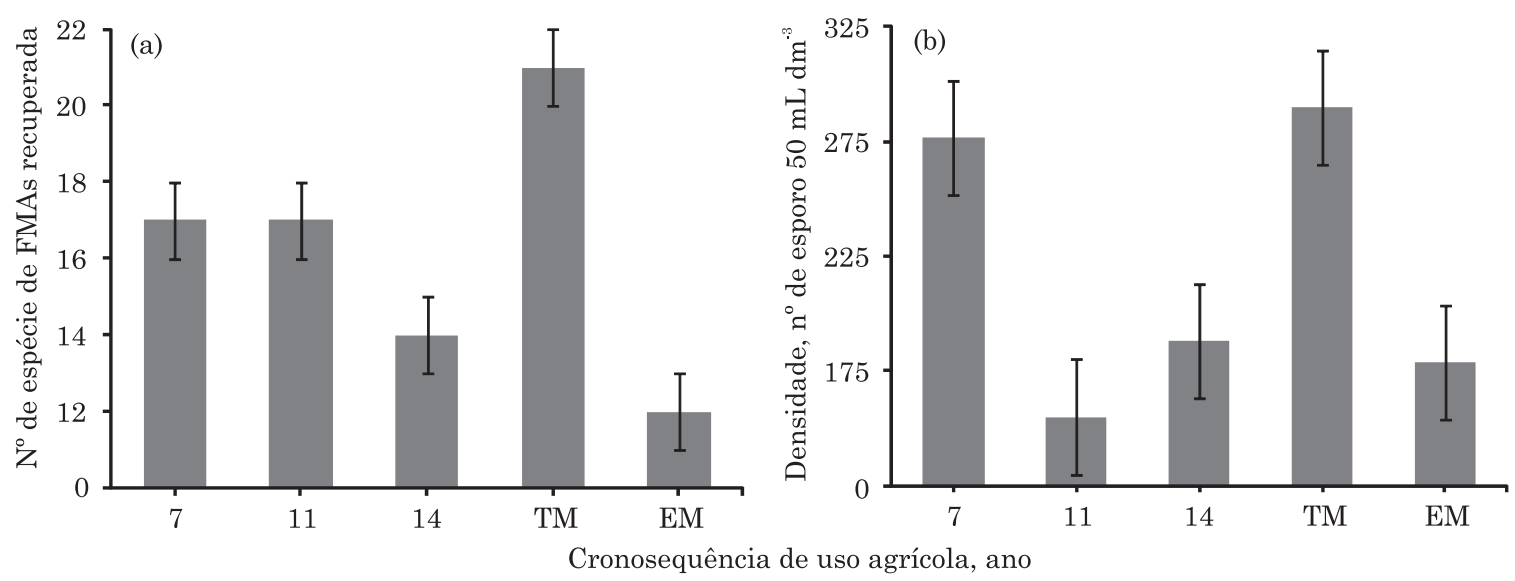

Figura 1. Número de espécies (a) e densidade de esporos (b) de FMAs nas áreas de uso agrícola (7, 11 e 14 anos) e sem alteração antrópica. TM: topo dos murundus; e EM: entre os murundus. Barras referem-se à DMS a $5 \%$ pelo teste de Scott-Knott.

pesquisas demonstrem sua importância para a sustentabilidade desse solo. Portanto, este estudo tratou-se do primeiro relato da ocorrência e da estrutura da comunidade de FMAs em fitofisionomia de campos de murundus, contribuiu para o maior entendimento dos FMAs no bioma Cerrado e demonstrou que as alterações promovidas pela conversão da área alteram a riqueza e ocorrência dos fungos micorrízicos arbusculares, podendo comprometer a sustentabilidade desse solo.

\section{CONCLUSÕES}

1. A conversão de campos de murundus em área agrícola modificou a ocorrência e composição da comunidade de fungos micorrízicos arbusculares.

2. A conversão das áreas de campos de murundus para agricultura promoveu a ocorrência de espécies de FMAs específicos; e a manutenção das espécies Acaulospora scrobiculata, Glomus macrocarpum, Claroideoglomus etunicatum e Gigaspora sp. ocorreram em todas as áreas e nos dois anos estudados.

3. A não ocorrência de algumas espécies nas áreas de cultivo representa perda de diversidade desses fungos, quando comparados às áreas de campo de murundus.

\section{AGRADECIMENTOS}

Ao proprietário da Fazenda Boa Vista, o qual permitiu a instalação deste estudo e pelo apoio logístico para a coleta de solo. Ao CNPq, pelo auxílio financeiro (processos 577559/2008-7 e 559682/2009-3) e pela bolsa de pesquisa (processos 308207/2011-4 e 302343/2012-1). À Capes, pela concessão da bolsa de mestrado à primeira autora.

\section{LITERATURA CITADA}

CARRENHO, R.; SILVA, E.S.; TRUFEM, S.F.B. \& BONONI, V.L.R. Successive cultivation of maize and agricultural practices on root colonization, number of spores and species of arbuscular mycorrhizal fungi. Braz. J. Microbiol., 32:262-270. 2001.

CARVALHO, F.; SOUZA, F.A.; CARRENHO, R.; MOREIRA, F.M.S.; JESUS, E.C. \& FERNANDES, G.W. The mosaic of habitats in the high-altitude Brazilian rupestrian fields is a hotspot for arbuscular mycorrhizal fungi. Appl. Soil Ecol., 52:9-19, 2012.

CARVALHO, L.M.; CACADOS, I. \& MARTIRIS-LOUCAO, M.A. Temporal and spatial variation of arbuscular mycorrhizas in salt marsh plants of Tagus estuary (Portugal). Mycorrhiza, 11:303-309, 2001.

CORDEIRO, M.A.S.; CARNEIRO, M.A.C.; PAULINO, H.B. \& SAGGIN-JÚNIOR, O.J. Colonização e densidade de esporos de fungos micorrízicos em dois solos do Cerrado sob diferentes sistemas de manejo. Pesq. Agropec. Trop., 35:147-153, 2005.

CORREIA, C.R.M.A.; REATTO, A.; MARTINS, É.S.; SILVA, E.M.; CALDAS, L.S. \& FAGG, J.F. Micorriza Arbuscular: Um bioindicador da fertilidade dos solos e da distribuição de árvores no Bioma Cerrado. Planaltina, Embrapa Cerrados, 2004. 169p. (Boletim de Desenvolvimento de Pesquisa, 132)

COX, G.W.; GAKAHU, C.G. \& WAITHAKA, J.M. The form and small stone content of large earth mounds constructed by mole rats and termites in Kenya. Pedobiologia, 33:307314, 1989.

ENTRY, J.A.; RYGIEWICZ, P.T.; WATRUD, L.S. \& DONNELLY, P.K. Influence of adverse soil conditions on the formation and function of arbuscular mycorrhizas. Adv. Environ. Res., 7:123-138, 2002.

ESCUDERO, V. \& MENDOZA, R. Seasonal variation of arbuscular mycorrhizal fungi in temperate grasslands along a wide hydrologic gradient. Mycorrhiza, 15:291-299, 2005. 
FERREIRA, D.A.; CARNEIRO M.A.C. \& SAGGIN, O.J.J. Fungos micorrízicos arbusculares em um Latossolo Vermelho de Cerrado sob diferentes manejos e uso. R. Bras. de Ci. Solo, 36:51-61, 2012.

FERREIRA, D.F. Sistema de análises de variância para dados balanceados. Lavras, Universidade Federal de Lavras, 2000. (SISVAR 4. 1. pacote computacional)

GERDEMANN, J.W. \& NICOLSON, T.H. Sopres of micorrhizal endoge species extracted from soil by wet sieving and decating. Trans. Brit. Mycol. Soc., 26:235-246, 1963.

GUADARRAMA-CHÁVEZ, P.; CAMARGO-RICALDE, S.L. \& CASTILLO-ARGÜEROS, S. Los hongos micorrizógenos arbusculares de la region de Nizanda, Oaxaca, México. Bol. Soc. Bot. México, 81:131-137, 2007.

JENKINS, W.R.A. Rapid centrifugal flotation technique for separating nematodes from soil. Plant Dis. Rep., 48:62, 1964.

KLIRONOMOS, J.N.; McCUNE, J.; HART, M. \& NEVILLE, J. The influence of arbuscular mycorrhizae on the relationship between plant diversity and productivity. Ecol. Lett., 3:137-141, 2000.

MANGAN, S.A.; EOM, A.H.; ADLER, G.H.; YAVITT, J.B. \& HERRE, E.A. Diversity of arbuscular mycorrhizal fungi across a fragmented forest in Panama: Insular spore communities differ from mainland communities. Oecologia, 141:687-700, 2004.

MARIMON, B.S.; MARIMON-JUNIOR, B.H.; MEWS, H.A.; JANCOSKI, H.S.; FRANCZAK, D.D.; LIMA, H.S.; LENZA, E.; ROSSETE, A.N. \& MORESCO, M.C. Florística dos campos de murundus do Pantanal do Araguaia, Mato Grosso, Brasil. Acta Bot. Bras., 26:181-196, 2012.

MIRANDA, J.C. \& MIRANDA, L.N. Micorriza arbuscular. In: VARGAS, M.A. \& HUNGRIA, M. ed. Biologia dos solos de Cerrado. Planaltina, Embrapa Cerrados, 1997. p.69-123.

MIRANDA, J.C.C. Cerrado: Micorriza arbuscular: ocorrência e manejo. Planaltina, Embrapa Cerrados, 2008. p.169.

MIRANDA, J.C.C. \& MIRANDA, L.N. Contribuição da micorriza arbuscular para a produtividade e sustentabilidade nos sistemas de produção com plantio direto no Cerrado. Planaltina, Embrapa Cerrados, 2007. 6p. (Boletim Técnico, 134)

MIRANDA, J.C.C.; VILELA, L. \& MIRANDA, L.N. Dinâmica e contribuição da micorriza arbuscular em sistemas de produção com rotação de culturas. Pesq. Agropec. Bras., 40:1005-1014, 2005.

MOLLARD, J.D. Landforms and surface materials of Canada: A stereoscopic airphoto atlas and glossary. Regina, Mollard \& Associates, 1982. 231p.

OLIVEIRA FILHO, A.T. The vegetation of Brazilian 'murundus' - the island-effect on the plant community. J. Trop. Ecol., 8:465-486, 1992.

RAHLAO, S.J.; HOFFMAN, M.T.; TODD, S.W. \& McGRATH, K. Long-term vegetation change in the Succulent Karoo, South Africa following 67 years of rest from grazing. J. Arid. Environ., 72:808-819, 2008.
RESENDE, M.; CURI, M.; RESENDE, S.B. \& CORREAA, G.F. Pedologia: Base para distinção de ambientes. 5.ed. Lavras, Universidade Federal de Lavras, 2007. 322p.

SIEVERDING, E. Vesicular-arbuscular mycorrhiza management in tropical agrosystems. Eschborn, Bremer Verlag, 1991. p.279-285.

SILVA, L.C.R.; STERNBERG, L.; HAIDAR, R.F. \& VALE, G.D. Deciphering earth mound origins in central Brazil. Plant Soil, 336:3-14, 2010.

SIQUEIRA, J.O.; COLOZZI-FILHO, A. \& OLIVEIRA, E. Ocorrência de micorrizas vesicular-arbuscular em agro e ecossistemas do Estado de Minas Gerais. Pesq. Agropec. Bras., 24:1499-1506, 1989.

SIQUEIRA, J.O.; ROCHA JÚNIOR, W.F.; OLIVEIRA, E. \& COLOZZI-FILHO, A. The relationship between vesicular-arbuscular mycorrhiza and lime: associated effects on the growth and nutrition of Brachiaria grass (Brachiaria decumbens). Biol. Fert. Soils, 10:65-71, 1990.

SOUZA, F.A.; STÜRMER, S.L.; CARRENHO, R. \& GOTO, B.T. Classificação e taxonomia de Fungos Micorrízicos Arbusculares e sua diversidade e ocorrência no Brasil. In: SIQUEIRA, J.O.; SOUZA, F.A.; CARDOSO, E.J.B.N. \& TSAI, S.M., eds. Micorrizas: 30 anos de pesquisas no Brasil. Lavras, Universidade Federal de Lavras, 2010. p.15-74.

SOUZA, D.M.G. \& LOBATO, E. Cerrado: Correção do solo e adubação. 2.ed. Brasília, Embrapa Informação Tecnológica, 2004. 416p.

SOUZA, F.A.; STÜRMER, S.L.; CARRENHO, R. \& GOTO, B.T. Classificação e taxonomia de Fungos Micorrízicos Arbusculares e sua diversidade e ocorrência no Brasil. In: SIQUEIRA, J.O.; SOUZA, F.A.; CARDOSO, E.J.B.N. \& TSAI, S.M., eds. Micorrizas: 30 anos de pesquisas no Brasil. Lavras, Universidade Federal de Lavras, 2010. p.15-74.

STEVENS, K.J.; WALL, C.B. \& JANSSEN, J.A. Effects of arbuscular mycorrhizal fungi on seedling growth and development of two wetland plants, Bidens frondosa L., and Eclipta prostrate (L) L., grown under three levels of water availability. Mycorrhiza, 21:279-278. 2011.

STÜRMER, S.L. \& SIQUEIRA, J.O. Diversity of arbuscular mycorrhizal fungi in Brazilian ecosystems. In: MOREIRA, F.M.S.; SIQUEIRA, J.O. \& BRUSSAARD, L., eds. Soil Biodiversity in Amazonian and other Brazilian ecosystems. London, CABI Publishing, 2006. p.206-236.

STÜRMER, S.L. \& SIQUEIRA, J.O. Species richness and spore abundance of arbuscular mycorrhizal fungi across distinct land uses in Western Brazilian Amazon. Mycorrhiza, 21:255-267, 2011.

van der HEIJDEN, M.G.A.; KLIRONOMOS, J.N.; URSIC, M.; MOUTOGLIS, P.; STREITWOLF-ENGEL, R.; BOLLER, T.; WIEMKEN, A. \& SANDERS, I.R. Mycorrhizal fungal diversity determines plant biodiversity, ecosystem variability and productivity. Nature, 396:69-72, 1998. 
WANG, Y.; QIU, Q.; YANG, Z.; HU, Z.; TAM, N.F.Y. \& XIN, G. Arbuscular mycorrhizal fungi in two mangroves in South China. Plant Soil, 331:181-191, 2010.

ZANGARO, W.; NISHIDATE, F.R.; VANDRESEN, J.; ANDRADE, G. \& NOGUEIRA, M.A. Root mycorrhizal colonization and plant responsiveness are related to root plasticity, soil fertility and successional status of native woody species in southern Brazil. J. Trop. Ecol., 23:53-62, 2007.
ZHANG, Y.; GUI, L, D. \& LIU, R.J. Survey of arbuscular mycorrhizal fungi in deforested and natural forest land in the subtropical region of Dujiangyan, southwest China. Plant Soil, 261:257-263, 2004.

ZHAO, Y.; DAI, X.; BLACKWELL, H.E.; SCHREIBER, S.L. \& CHORY, J. SIR1, an upstream component in auxin signaling identified by chemical genetics. Science, 301:1107-1110, 2003. 